\title{
DIAGNOSTIC APPROACH TO THE INCORRECT POSITION OF LOWER SECOND PREMOLARS
}

\author{
Zornica Valcheva ${ }^{1}$, Hristina Arnautska ${ }^{1}$, Tsvetan Tonchev ${ }^{2}$, Tihomir Georgiev ${ }^{2}$ \\ ${ }^{1}$ Department of Prosthetic Dentistry and Orthodontics, Faculty of Dental Medicine, \\ Medical University of Varna \\ ${ }^{2}$ Department of Oral and Maxillofacial Surgery and Special Imaging Diagnostics, \\ Faculty of Dental Medicine, Medical University of Varna
}

\begin{abstract}
The lower second premolar is regarded as the third most common impacted tooth after lower wisdom teeth and upper canines. The aim of the present study is to apply objective diagnostic methods to help determine the impaction likelihood of lower second premolars and their distal inclination. For the purpose of this study 137 panoramic radiographs (OPGs) have been examined of children aged 8-16 years. The methodology used in this study helped measure the inclination and angle between the lower second premolar and the crown and axis of the first permanent molar and the mandibular plane. Prevalence of the distal inclination of lower second premolars was observed. Indicators for the impacted lower second premolar proved to be the germ inclination of the lower fifth tooth in relation to the first molar of more than $30^{\circ}$ and the intersection of the crown of the sixth tooth by the axis of premolar as well as the inclination towards the mandibular plane of less than $68^{\circ}$. Early extraction of deciduous molars, reserving or creating space when there is a lack of space, allow for favourable conditions for altering the eruption path of the premolar.
\end{abstract}

Keywords: lower premolar, second temporary molar, impacted premolar, disturbed eruption path

\section{INTRODUCTION}

The lower second premolar is regarded as the third most common impacted tooth after lower wisdom teeth and upper canines. Impaction of the mandibular fifth teeth accounts for $24 \%$ of all impacted teeth, apart from wisdom teeth. $(1,2)$ The prevalence of impacted premolars vary according to age; with the elderly population the overall frequency being $0.5 \%$ (of which for lower second premolar it has been found to range from 0.2 to $0.3 \%$ ) (3-5).

Address for correspondence:

Hristina Arnautska

Department of PDM and Orthodontics

Faculty of Dental Medicine

Medical University - Varna

84 Tzar Osvoboditel Blvd.

9000 Varna

e-mail: tineia@yahoo.com

Received: October 29, 2015

Accepted: December 1, 2015
In the early stage of its development it is not uncommon for the lower second premolar to appear close to the inferior border of the mandible, which can be easily observed on a panoramic radiograph (OPG). Typically the eruption path of the tooth follows the direction/path of resorption of the root of the preceding temporary molar. However, sometimes the tooth germ alters its position and tilts from its normal position, making tooth eruption difficult, and in some cases may even lead to its impaction. Without further treatment the tooth may be retained in the jaw with the risk of damaging adjacent teeth and/or developing cyst formations.

The causes of impaction of lower fifth teeth are due to local, etiologic and genetic factors. The most common reason proves to be the lack of space and the ectopic position of the tooth germ of the second premolar (6-9). Literature rarely reports etiologic factors to be odontomas, supernumerary and ankylosed teeth (10). A number of authors consider impaction 
part of a genetically determined pattern of development of dental anomalies $(11,12)$. These include hypodontia, particularly of the opposing second premolar (9), size reduction of the teeth, microdontia of the lateral incisor, impacted upper canines, dental transposition. Literature also lists causes such as root dilaceration, dysostosis, fibrosis and trauma (in $<1 \%$ of the cases).

More commonly, the incorrect position of the lower second premolar is seen in its distal inclination: for tooth germs to about $56.5 \%$ and only to $25 \%$ with mesial inclination (13).

Literature reports cases of early eruption of the sixth teeth, causing trauma in the periodontal tissues of the second temporary molar, resulting in ankylosis of the latter, its melting and movement down towards the germ of the second premolar, sometimes completely below the alveolar ridge (the so-called primary impaction) $(14,15)$. Consequently, ankylosis hampers the normal eruption of the permanent tooth and the germ alters its direction.

AIM of the present study is to apply objective diagnostic methods for determining impaction likelihood of lower second premolars and their distal inclination.

\section{MATERIALS AND METHODS}

For the purpose of this study 137 panoramic radiographs (OPGs) and situation models have been examined of children aged 8 - 16 years, available from the database of the Department of Orthodontics at the Faculty of Dental Medicine of Medical University - Varna.

The long axes of the first molars and the germs of the second premolars were drawn on the panoramic radiographs under research and the angle between their axes was estimated. The methodology of Becker et al. (16) was used to observe the angle of the germ axis of the second lower premolar and mesial surface of the crown of the first permanent molar. The cases where the long axis of the fifth tooth germ did not intercept the mesial surface of the sixth tooth were considered favourable and were expressed as a plus $(+)$, i.e. normal eruption was to be expected. When intersection of the long axes of both teeth took place within the permanent molar, the eruption prognosis was considered unfavourable and was expressed as a minus (-) (Fig. 1). The angles between the long axes of
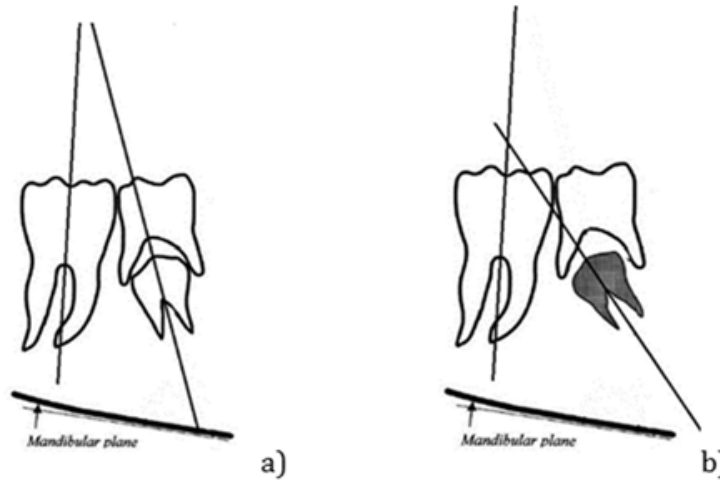

Figure 1. Methodology of Becker et al. (16): a) favourable prognosis (+) b) unfavourable prognosis (-)

the first molar and second premolar in the lower jaw were also drawn, namely the $\gamma$ - angle as per Baccetti et al.'s methodology (17). Measurements were taken of the $\theta$-angle as per Shalish et al. (Fig. 2) (23), formed by the long axis of the fifth tooth and the line drawn tangent to the inferior border of the mandible on the radiograph. According to the authors the values of these two angles for normal eruption range as follows: for $\gamma$-angle $8.4 \pm 9.9^{\circ}$ and for $\theta$-angle $82.3 \pm 12.8^{\circ}$.

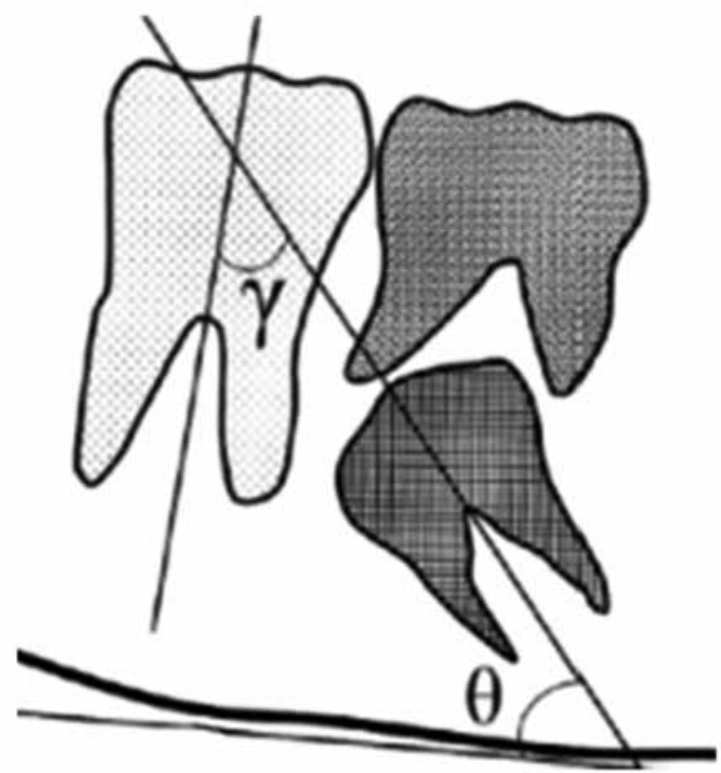

Figure 2. Methodology for determining the germ inclination of permanent fifth tooth in relation to the axis of the first molar ( $\gamma$-angle as per Baccetti et al.) and the mandibular plane ( $\theta$-angle as per Shalish et al.) 


\section{RESULTS AND DISCUSSION}

137 OPGs were explored in this study, however, the examination of 36 radiographs (26.3\%) revealed impaction likelihood of second premolars, i.e. unfavourable prognosis. Out of all teeth observed having unfavourable position, 27 radiographs displayed impaction likelihood of second premolar in the lower jaw whereas 9 radiographs showed their impaction

\section{Impacted Lower Premolars}

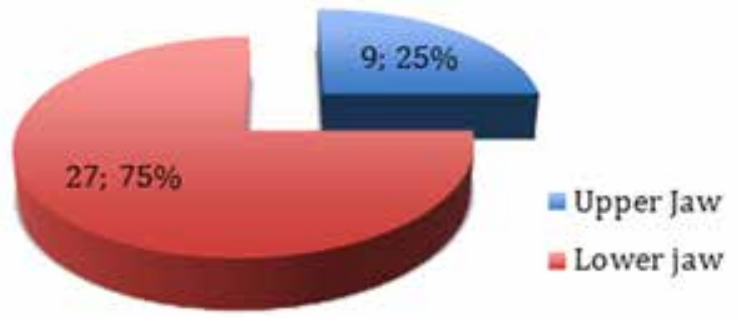

Figure 3. Impaction incidences for upper and lower jaw

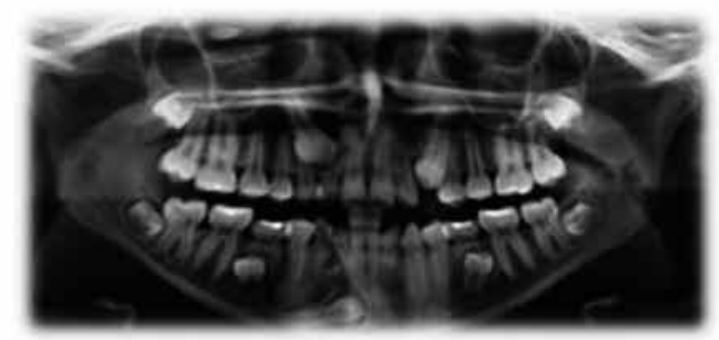

- palatal position of upper canine;

- infraposition of second molars;

- a cyst and a retained lower canine;

- odontoma;

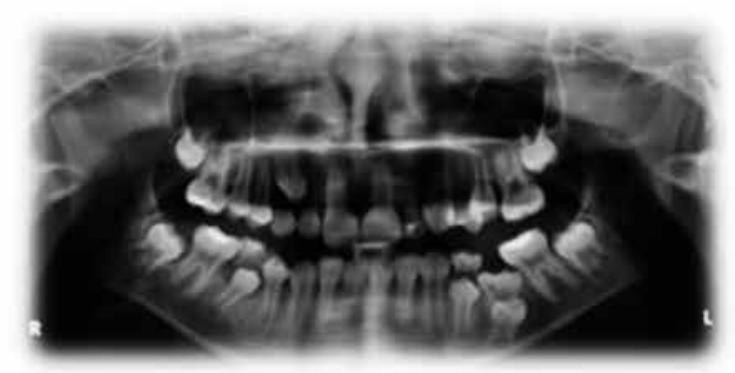

- ankylosis and mesial inclination of first molar hypodontia likelihood in the upper jaw (Fig. 3). The conclusion follows that there is a higher incidence of impaction of lower premolars compared to the upper jaw. Etiologic causes are probably linked to the greater density of the lower jaw as well as to genetic components.

Within the study group the analyses of the clinical picture disclosed the following etiologic factors leading to impaction of premolars in the lower jaw:

- Delayed root resorption of the temporary tooth and its retention in the dental arch, the result of a slow eruption tendency of more than a year (7 cases)

* Closing of the space for the lower second premolar due to medialisation of adjacent teeth due to premature loss of temporary molars (for 6 teeth)

* Mesial inclination of the first molar, the result of untreated approximal caries (6 cases)

* Ankylosis of temporary teeth (1 case)

* Follicular cysts (1 case)

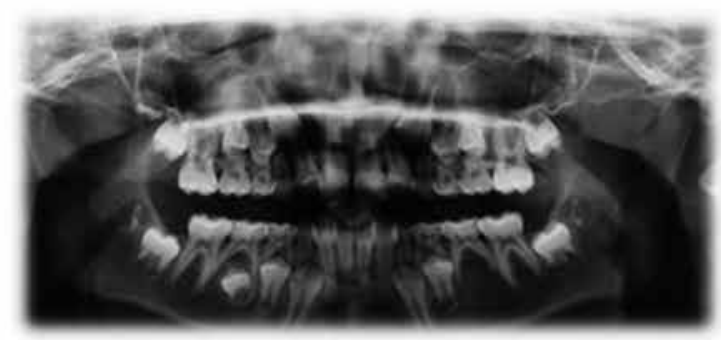

- hypodontia on the opposite side;

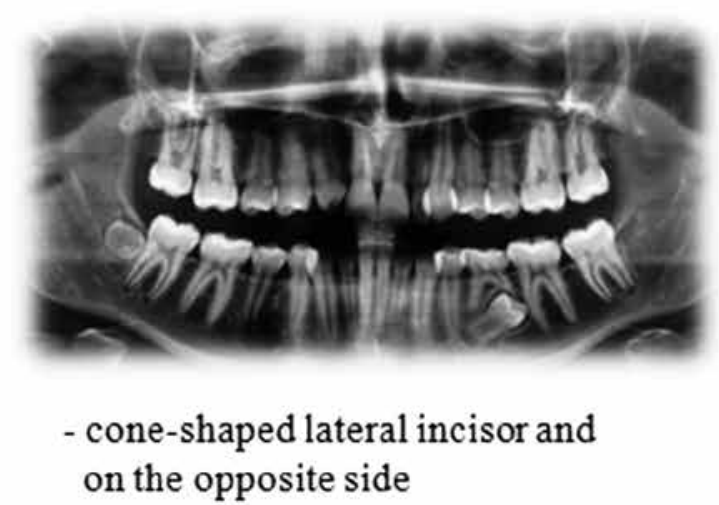

Figure 4. Etiologic factors leading to impaction of premolars 
* Inflammation of the tissues surrounding the root of the temporary tooth, the result of caries treatment and subsequent complications (4 cases)

* Ectopic position of the germ of the permanent tooth (11 cases considered genetically determined, with the presence of hypodontia, abnormal shape of lateral incisors, overall impaired development, etc.

The etiologic factors analysed in our study coincided with findings observed by other authors (1922). This confirms the significance of genetic and local factors and their differentiation as causes of impacted second premolars.

The direction of inclination of lower fifth teeth was also explored. The distal inclination of the germ of fifth permanent teeth shows prevalence for 33 teeth (91.6\% of the cases studied). Only three teeth had mesial inclination of the germ in the upper jaw.

In the cases for lower jaw, analyses were performed of the $\gamma$-angle between the long axis of the sixth tooth and the axis of the germ of the lower sec-

Table 1. Distribution of mean values of $\gamma$-angle for the
study and clinical group
\begin{tabular}{|ccc|c|}
\hline Angle $\gamma$ & $\mathrm{N}$ & Mean & Range \\
\hline Impacted P2 & 27 & $40^{\circ}$ & $30^{\circ}-56^{\circ}$ \\
Normally erupting P2 & 45 & $12^{\circ}$ & $8^{\circ}-20^{\circ}$ \\
\hline
\end{tabular}

ond premolar where there was impaction likelihood. The angle for normally erupting premolars on the opposite side was also measured in the control group

The results are given in Table 1:

As shown in Table 1, in the cases of fifth teeth retention the $\gamma$-angle has a mean value of $40^{\circ}$ (ranging from $30^{\circ}$ to $56^{\circ}$ ). Within the control group the values of the $\gamma$-angle seem to range from $8^{\circ}$ to $20^{\circ}$ (mean value: $12^{\circ}$ ) which is closer to the result obtained by Baccetti et al. (17). The difference in values between the study group with impacted fifth teeth and the control group is statistically significant $(\mathrm{P}<0.05)$. The mean value of $\gamma$-angle in the clinical group significantly exceeds the one published by Baccetti et al. (17) for the inclination values of the impacted lower fifth tooth $\left(21.7^{\circ} \pm 19.4^{\circ}\right)$. Hence, it can be assumed that if the germ inclination of the fifth tooth in the lower jaw in relation to the first molar exceeds $25^{\circ}$, preventive measures ought to take place in order to create conditions for straightening the straightening the axis of the premolar, making way for its root and subsequent eruption.

In the present study values such as $20^{\circ}-30^{\circ}$ are considered border-line cases and are debatable since the long axis of the fifth tooth germ appears tangent to the mesial surface of the sixth tooth, which points to a border-line favourable prognosis where there is chance to correct the eruption path of the second premolar. The values of the $\theta$-angle were also compared, $\theta$-angle being the angle formed between the

Table 2. Distribution of mean values of $\theta$-angle for the
study and clinical group
\begin{tabular}{|l|ccc|}
\hline Angle $\theta$ & N & Mean & Range \\
\hline Impacted P2 & 27 & $68^{\circ}$ & $51^{\circ}-90^{\circ}$ \\
Normally erupting P2 & 45 & $83.2^{\circ}$ & $66^{\circ}-94^{\circ}$ \\
\hline
\end{tabular}

long axis of the fifth tooth germ and the tangent to the inferior border of the mandible.

The results are given in Table 2:

As shown in Table 2, in the cases of likely impaction of fifth teeth the mean inclination of the axis of the second premolar to the mandibular plane is $68^{\circ}$, which is close to the results obtained by Baccetti et al. $(13,17,23)$. For normally erupting fifth teeth the value of this angle is $83^{\circ}$, which coincides with the results of other authors (17) $\left(82.3^{\circ} \pm 12.8\right.$ as per authors). The difference in values between the two groups is statistically significant $(\mathrm{P}<0.05)$.

The etiology of impacted premolars is extremely diverse. The role of the genetic mechanism in the impaired eruption of fifth teeth is undeniable. The presence of hypodontia, microdontia of lateral incisors, impacted canines and other genetically related disorders should only point to the increasing inclination of the lower fifth teeth. Among local factors of greatest importance is the mesial inclination of the sixth teeth as well as the loss of space due to dental caries. Malposition of premolars appears much more frequent alongside other dental abnormalities rather than as an inclination alone.

The present study reveals more incidences of retention of lower rather than upper second premolars 
with significant prevalence towards distal inclination of lower premolars of over $30^{\circ}$. The increase in value of the angle between the long axis of the first permanent molar and the germ of the fifth tooth and the reduction of the angle between the long axis of the premolar and the tangent to the lower jaw increases the likelihood of impacted premolar.

\section{CONCLUSION}

Early detection of impaction likelihood of lower second premolars is important for prevention purposes. Timely diagnosis will lead to early correction and treatment prior to their complete root development, which in turn will give a favourable prognosis. The inclination of the fifth tooth axis in relation to the first permanent molar of over $30^{\circ}$ and the intersection of the crown of the sixth tooth by the axis of the premolar are indicative of impaction. Early extraction of deciduous molars, reserving or creating space when there is a lack of space, allow for favourable conditions for altering the eruption path of the premolar.

\section{REFERENCES}

1. Collett AR. Conservative management of lower second premolar impaction. Aust Dent J. 2000 Dec;45(4):279-81.

2. Thilander H, Thilander B, Persson G. Treatment of impacted teeth by surgical exposure. A survey study. Sven Tandlak Tidskr1973; 66:519-525.

3. Andreasen JO. Textbook and color atlas of tooth impactions. Diagnosis, treatment and prevention. Copenhagen: Munksgaard; 1997, 177-195.

4. Tsukamoto S, Braham RL. Unerupted second primary molar positioned inferior to the second premolar: clinical report. ASDG J Dent CHILD. 1986; 53:67-9.

5. Borsatto M, Sant'Anna A, Niero H, et al. Unerupted second primary mandibular molar positioned inferior to the second premolar: case report. Pediatr Dent. 1999; 21:205-8.

6. Kudo M, Konta K, Oguchi H. Clinical Observation of Unerupted Primary Molars in the Patients of Hokkaido University Pediatric Dental Clinic. Jpn J Ped Dent. 1996; 34:824-34.

7. Bateman R, Emmering TE. Deciduous impaction. Oral Surg. 1976; 42:852-3.
8. Park JK. Submerged impacted primary molar. Oral Surg. 1979; 48:383.

9. Bhasker SN. Orban's Oral Histology and Embryology. St. Louis: Mosby; 1986, 372-3.

10. Chida R, Chiba K, Saitoh T, Mayanagi H. A case for impacted second deciduous molar caused by anomalies in the position of the tooth germ of succeeding permanent tooth. Jpn J Ped Dent, 1982; 20:7480. Japanese.

11. Haavikko K. The formation and the alveolar and clinical eruption of the permanent teeth. An orthopantomographic study. Suom Hammaslaak Toim. 1970; 66:103-70.

12. Ooe $\mathrm{T}$. Changes of positions of deciduous molar and premolar germs during development. Okajimas Folia Anat Jpn. 1968; 44:83-97.

13. Wasserstein A, BrezniakN, ShalishM, HellerM, and Rakocz M.Angular Changes and Their Rates in Concurrence to Developmental Stages of the Mandibular Second Premolar. The Angle Orthodontist. 2004; 74(3):332-36.

14. Bianchi SD, Roccuzzo M. Primary impaction of primary teeth: a review and report of three cases. J Clin Pediatric Dent, 1991; 15:165-8.

15. McNamara B, McNamara TG. Mandibular Premolar Impaction: 2 Case Reports Clare. JCDA. December 2005/January 2006; 71(11):859-63.

16. Becker A. The orthodontic treatment of impacted teeth. New York: Thieme; 1998. 234.

17. Baccetti T, Leonardi M, Giuntini V. Distally displaced premolars: A dental anomaly associated with palatallydisplaced canines, Am J Orthod Dentofacial Orthop. 2010; 138(3):318-322.

18. Burch J, Ngan P, Hackman A. Diagnosis and treatment planning for unerupted premolars. Pediatr Dent, 1994 Mar-Apr; 16(2):89-95.

19. Konstat MM, White GE. Ankylosed teeth: a review of the literature, J Mass Dent Soc 1975; 24:74-8.

20. Biederman W. The problem of the ankylosed tooth. Dent Clin North Am, 1968 Jul: 409 -24.

21. Andlaw RJ. Submerged deciduous molars: a review, with special reference to the rationale of treatment. J Int Assoc Dent Child, 1974; 5:59-66.

22. Shalish M, Peck Sh, Wasserstein A, Peck L. Increased Occurrence of Dental Anomalies Associated with Infraocclusion of Deciduous Molars, Angle Orthod. 2010 May; 80(3):440-5. 